\title{
Recent advances in the pharmacological management of migraine
}

\author{
Julio Pascual
}

Address: Service of Neurology, University Hospital Marqués de Valdecilla, Av Valdecilla s/n, 39008 Santander, Spain

Email: juliopascual@telefonica.net

Fl000 Medicine Reports 2009, I:39 (doi:10.3410/MI-39)

The electronic version of this article is the complete one and can be found at: http://FI000.com/Reports/Medicine/content/I/39

\begin{abstract}
Pharmacological treatment of migraine includes acute and, in some patients, preventive medications. Here, the most relevant recent advances in migraine management are reviewed. Regarding acute treatment, new data indicate that early treatment of migraine attacks should be recommended to those patients who do not show a clear response when pain is already moderate or severe. It has also been shown that, in terms of efficacy, the combination of a nonsteroidal anti-inflammatory drug plus a triptan is superior to monotherapy. In the immediate future it seems that a new class, the antagonists of calcitonin gene-related peptide, will offer at least the same efficacy as that of potent triptans, but a much better tolerability and no vascular contraindications. Concerning migraine prevention, news has been concentrated on the management of chronic migraine, with the appearance of guidelines for clinical trials in chronic migraine and the demonstration that it is a treatable entity, even in the presence of overuse criteria.
\end{abstract}

\section{Introduction and context}

Migraine is a highly prevalent and disabling illness. Globally, $11-13 \%$ of the adult population suffers from active migraine. Optimal pharmacological treatment is therefore crucial so the patient can have a reasonable quality of life. Pharmacological treatment of migraine includes acute symptomatic approaches, which are needed by all migraineurs, as well as preventatives, which should be prescribed in at least one-quarter of migraine patients.

Recommended current symptomatic treatment of migraine encompasses the judicious use of nonsteroidal anti-inflammatory drugs (NSAIDs) and the 5-hydroxytryptamine $(5-\mathrm{HT} 1 \mathrm{~B} / 1 \mathrm{D})$ selective receptor agonists known as 'triptans'. Simple/combined analgesics and ergotamine-containing medications are not routinely recommended in this indication because, compared with NSAIDs and triptans, they are less efficacious, show a poorer tolerability (ergotics), and are prone to induce rebound headache and medication overuse [1]. At least $30 \%$ of migraine patients, however, either do not respond or show contraindications (for example, vascular risk factors) to the use of NSAIDs and/or triptans. Migraine patients with one or more attack per week need preventive treatment and, in addition, if attacks cause disability on 2 or more days per month and are not prevented by optimized acute treatment, preventive treatment is indicated if the patient is willing to take daily medication [2,3]. Options with a demonstrated efficacy in this indication include some beta-blockers, flunarizine, possibly low doses of amitriptyline, and the antiepileptics valproic acid and topiramate. In line with symptomatic treatment, no more than $60 \%$ of migraine patients respond to and tolerate preventatives. In conclusion, there is a need for better options for the symptomatic and preventative treatment of migraine. The most relevant recent advances for the management of migraine are reviewed here.

\section{Recent advances \\ Developments in acute treatment}

As mentioned above, at least $30 \%$ of patients do not respond to NSAIDs and/or triptans. How could the efficacy of symptomatic treatment be improved? Of course, an obvious approach is to explore nonoral routes 
of administration such as intranasal (with zolmitriptan $5 \mathrm{mg}$ or sumatriptan $20 \mathrm{mg}$ ) or even parenteral (for example, subcutaneous sumatriptan or intramuscular/ intravenous NSAIDs) routes, which have demonstrated a higher efficacy (but also a higher degree of adverse events) in migraine patients refractory to the oral route. As most migraine patients prefer the oral route, a few treatment approaches have been tested to try to improve the efficacy of current medications through this route [4].

Many trials have explored the efficacy of early treatment of migraine attacks (when pain is still mild) with triptans. In general, early treatment when pain was still mild was numerically better as compared with late treatment. The results of these trials, however, have been very controversial because designs were not homogeneous, the advantage in terms of therapeutic gain was not so clear, and early treatment could, at least theoretically, induce medication overuse [5]. A recent clinical trial has examined the efficacy of an oral triptan (almotriptan $12.5 \mathrm{mg}$ ) in the treatment of migraine in controlled conditions. This trial is interesting as, in the early treatment arm, patients were asked to treat migraine attacks when pain was still mild and by 1 hour of headache onset in order to test the Burstein allodynia hypothesis (that is, 1 hour after the beginning of migraine attack, allodynia develops and migraine becomes intractable). Globally, the results of this clinical trial suggest that treatment with almotriptan while migraine is still mild provides a clinically relevant enhancement in efficacy compared with treatment when pain has reached higher severity levels. Interestingly, the presence of allodynia did not correlate with treatment response, which suggests that allodynia is just one symptom associated with the migraine attack, such as phono/photophobia, and not a specific marker for response to acute treatment [6].

The second explored option to optimize acute migraine treatment in refractory patients is the combination of NSAIDs and triptans. A recent controlled trial with four arms (placebo, naproxen $500 \mathrm{mg}$, sumatriptan $85 \mathrm{mg}$, and both naproxen $500 \mathrm{mg}$ plus sumatriptan $85 \mathrm{mg}$ ) has shown interesting results with implications for clinical practice [7] (Figure 1). First, the two active medications were significantly more efficacious than placebo. Second, for all efficacy endpoints, there was at least a numerical advantage (not always significant) for sumatriptan as compared with naproxen. Even though the dose of naproxen could be considered too low for a fair comparison, these data suggest some superiority of triptans over NSAIDs (usually seen in clinical practice but undemonstrated in serious clinical trials). Finally, the combination of the two active medications was
Figure I. Summary of results of the controlled trial comparing naproxen $500 \mathrm{mg}$, sumatriptan $85 \mathrm{mg}$, and the combination of naproxen and sumatriptan

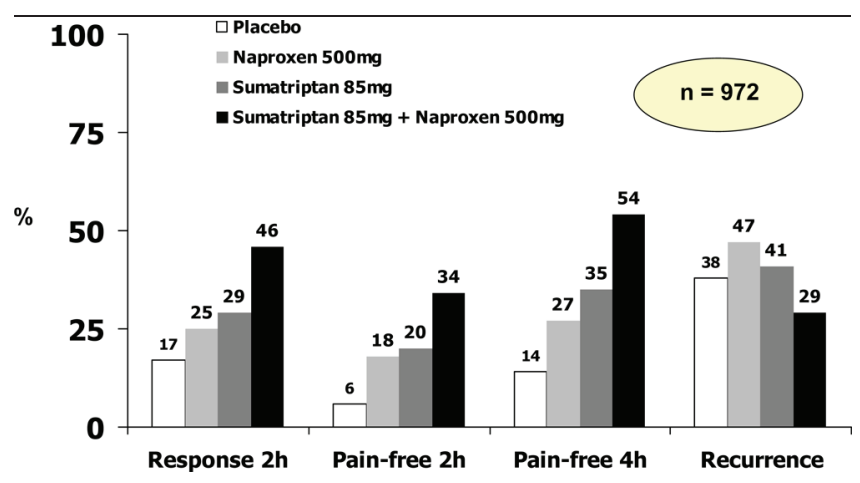

Note that each of the two medications as monotherapy is superior to placebo, and note also the numerical advantage of sumatriptan $85 \mathrm{mg}$ versus naproxen $500 \mathrm{mg}$ and the higher efficacy of the combination. Data are taken from [7].

clearly more efficacious than monotherapy. This indicates that combining the two active medications with different mechanisms of action translates in synergistic actions.

In spite of early treatment and combination approaches, there are still patients who do not respond or for whom triptans cannot be administered due to the presence of vascular contraindications, such as ischemic cardiomyopathy. In the last year, the first results with an oral compound of a totally new class of drug for the acute treatment of migraine, the antagonists of the calcitonin gene-related peptide (CGRP), have become available. These drugs act through a novel mechanism by blocking the action of CGRP on the CGRP receptor complex. CGRP is released in the extracerebral circulation during migraine attacks as a consequence of trigeminovascular activation. The first oral CGRP antagonist, telcagepant, has been shown to be as effective as two very efficacious triptans, zolmitriptan $5 \mathrm{mg}$ and rizatriptan $10 \mathrm{mg}$, for the acute treatment of migraine. The rates for adverse events, including chest symptoms, with telcagepant were comparable to those of placebo and lower than those seen for the two active triptans [8-10].

\section{Developments in preventive treatment}

Preventive treatment of migraine is indicated in patients with one or more attacks per week. Beta-blockers continue to be the medication of choice for those patients with migraine without aura attacks. Topiramate has been extensively studied in this indication and is now first in line for patients suffering from both migraine with and without aura attacks, especially if 
Figure 2. Reduction in migraine frequency in patients with chronic migraine with analgesic overuse

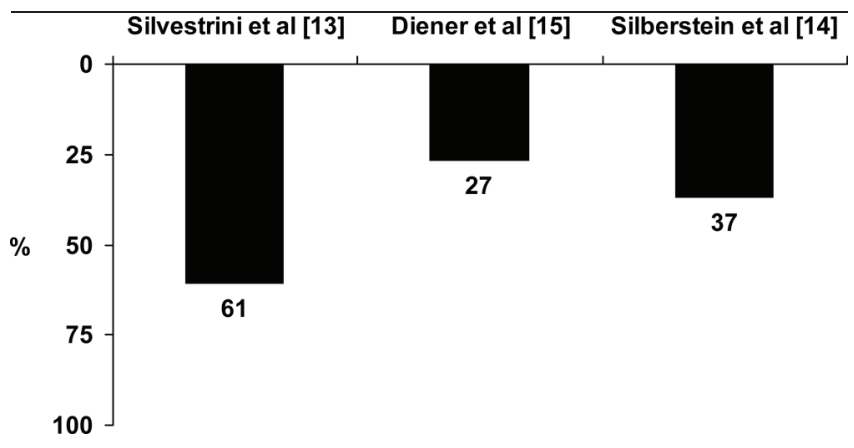

Data are taken from double-blind topiramate studies in [13-15].

they are obese, and the second option for patients who have not responded or show contraindications to betablockers. Flunarizine (available in some countries) or valproate are indicated if both beta-blockers and topiramate do not work. Not all antiepileptics work in migraine prevention, as shown by a recent negative double-blind controlled trial with oxcarbazepine [11].

In migraine prevention, the advances have been concentrated in the field of chronic migraine (migraine patients with headache more than 2 weeks per month). These patients had been excluded from classical clinical trials. This is incredible, as prevalence of chronic migraine is $2 \%$ in the general population, and it accounts for more than one-third of consultations in headache clinics. Guidelines for clinical trials in chronic migraine have recently been approved by the International Headache Society [12]. Very importantly, several trials with topiramate and, in the preliminary stages, with botulinum toxin have demonstrated that chronic migraine patients are treatable, even in the presence of analgesic overuse criteria [13-16] (Figure 2). In least in half of the patients with frequent attacks, further trials have shown that preventive treatment should be given for periods clearly longer than 6 months $[17,18]$. Finally, the concept of combination therapy with betablockers and topiramate in refractory patients looking for a synergistic action (as mentioned above for acute treatment) has also been explored with preliminary positive results [19].

\section{Implications for clinical practice Implications for acute treatment}

From the available results, it seems sensible to recommend early treatment of attacks (as soon as the patient is sure that he or she is having a migraine attack) with triptans at least in those patients who do not show a clear response when pain is already moderate or severe.
Preventive treatment must be considered in those patients who exceed the maximum recommendation of 10 triptan days per month [1].

Research investigating the combination of NSAIDs and triptans to optimize acute migraine treatment in refractory patients has indicated that treatment from the beginning of an attack with both an NSAID and a triptan should be recommended for those patients who do not exhibit a clear response with the two medications in monotherapy.

A new class of drug, the antagonists of the CGRP, will become available shortly as a new potential option for patients who do not respond or on whom triptans cannot be administered due to the presence of vascular contraindications. As this new therapeutic class of drugs is theoretically devoid of vasoconstrictor properties, they could mark a new era in migraine treatment. Interestingly, given the high potency of telcagepant, data from dose-finding studies suggest that central actions are necessary for the action of CGRP antagonists, which would also change our mind regarding the mode of action of acute antimigraine compounds [10].

\section{Implications for preventive treatment}

Beta-blockers continue to be the medication of choice to prevent migraine in patients without aura attacks, and the recent research into topiramate has indicated that it is first in line for patients suffering from both migraine with and without aura attacks, especially when they are obese or where beta-blockers are not appropriate. Extensive research on refractory patients suffering from chronic migraine has been carried out over the last few years. From a practical standpoint, the recent approval of the guidelines for clinical trials in chronic migraine is very important for the development of future management strategies all over the world. Even though chronic migraine remains a therapeutic challenge and overuse prevention must continue to be a high priority, recent work has shown that chronic migraine can be a treatable condition, even in the presence of analgesic overuse, as shown by the trials carried out with topiramate or combining preventatives such as beta-blockers and topiramate. There is hope that current trials and ongoing research will continue to shed light on this area and produce further clinical advances, such as the final demonstration of a role for botulinum toxin type A in the management of this condition.

\section{Abbreviations}

CGRP, calcitonin gene-related peptide; HT1B/1D, 5hydroxytryptamine 1B/1D; NSAID, nonsteroidal antiinflammatory drug. 


\section{Competing interests}

The author is on the advisory boards of Allergan, Inc and Merck Sharp \& Dohme Ltd.

\section{References}

I. Bigal ME, Lipton RB: Excessive acute migraine medication use and migraine progression. Neurology 2008, 71:1821-8.

2. Members of the task force: Evers S, Afra J, Frese A, Goadsby PJ, Linde M, May A, Sándor PS: EFNS guidelines on drug treatment of migraine. Report of an EFNS task force. Eur J Neurol 2006, I 3:560-72.

3. Steiner TJ, Paemeleire K, Jensen R, Valade D, Savi L, Lainez MJ, Diener HC, Martelletti P, Couturier EG; European Headache Federation; Lifting The Burden: The Global Campaign to Reduce the Burden of Headache Worldwide; World Health Organization: European principles of management of common headache disorders in primary care. J Headache Pain 2007, 8 (Suppl I):3-47.

4. Dowson AJ, Mathew NT, Pascual J: Review of clinical trials using early acute intervention with oral triptans for migraine management. Int J Clin Pract 2006, 60:698-706.

5. Ferrari MD: Should we advise patients to treat migraine attacks early? Cephalalgia 2004, 24:915-7.

6. Goadsby PJ, Zanchin G, Geraud G, de Klippel N, Diaz-Insa S, Gobel H, Cunha L, Ivanoff N, Falques M, Fortea J: Early vs. non-early intervention in acute migraine-'Act when Mild (AwM)'. A double-blind, placebo-controlled trial of almotriptan. Cephalalgia 2008, 28:383-91.

7. Brandes JL, Kudrow D, Stark SR, O'Carroll CP, Adelman JU, O'Donnell FJ, Alexander WJ, Spruill SE, Barrett PS, Lener SE: Sumatriptan-naproxen for acute treatment of migraine: a randomized trial. JAMA 2007, 297:|443-54.

8. Ho TW, Mannix LK, Fan X, Assaid C, Furtek C, Jones CJ, Lines CR, Rapoport AM; MK-0974 Protocol 004 study group: Randomized controlled trial of an oral CGRP receptor antagonist, MK-0974, in acute treatment of migraine. Neurology 2008, 70:1304-12.

9. Ho TW, Ferrari MD, Dodick DW, Galet V, Kost J, Fan X, Leibensperger $H$, Froman S, Assaid C, Lines C, Koppen H, Winner PK: Efficacy and tolerability of MK-0974 (telcagepant), a new oral antagonist of calcitonin gene-related peptide receptor, compared with zolmitriptan for acute migraine: a randomised, placebo-controlled, parallel-treatment trial. Lancet 2008, 372:2115-23.

FI000 Factor 6.0 Must Read

Evaluated by Peter Sándor 17 Dec 2008

10. Edvinsson L: CGRP-receptor antagonism in migraine treatment. Lancet 2008, 372:2089-90.
II. Silberstein S, Saper J, Berenson F, Somogyi M, McCague K, D’Souza J: Oxcarbazepine in migraine headache: a double-blind, randomised, placebo-controlled study. Neurology 2008, 70:548-55.

Changes Clinical Practice

FI000 Factor 3.0 Recommended

Evaluated by Lawrence Newman 15 Jul 2008

12. Silberstein S, Tfelt-Hansen P, Dodick DW, Limmroth V, Lipton RB, Pascual J, Wang SJ; Task Force of the International Headache Society Clinical Trials Subcommittee: Guidelines for controlled trials of prophylactic treatment of chronic migraine in adults. Cephalalgia 2008, 28:484-95.

13. Silvestrini M, Bartolini M, Coccia M, Baruffaldi R, Taffi R, Provinciali L: Topiramate in the treatment of chronic migraine. Cephalalgia 2003, 23:820-4.

14. Silberstein SD, Lipton RB, Dodick DW, Freitag FG, Ramadan N, Mathew N, Brandes JL, Bigal M, Saper J, Ascher S, Jordan DM, Greenberg SJ, Hulihan J; Topiramate Chronic Migraine Study Group: Efficacy and safety of topiramate for the treatment of chronic migraine: a randomised, double-blind, placebo-controlled trial. Headache 2007, 47:170-80.

15. Diener HC, Bussone G, Van Oene JC, Lahaye M, Schwalen S, Goadsby PJ; TOPMAT-MIG-20I(TOP-CHROME) Study Group: Topiramate reduces headache days in chronic migraine: a randomised, double-blind, placebo-controlled study. Cephalalgia 2007, 27:814-23.

FI000 Factor 6.0 Must Read

Evaluated by Manjit Matharu 04 Mar 2008

16. Dodick DW, Mauskop A, Elkind AH, DeGryse R, Brin MF, Silberstein SD: Botulinum toxin type $\mathbf{A}$ for the prophylaxis of chronic daily headache: subgroup analysis of patients not receiving other prophylactic medications: a randomized double-blind, placebo-controlled study. Headache 2005, 45:315-24.

17. Diener HC, Agosti R, Allais G, Bergmans P, Bussone G, Davies B, Ertas M, Lanteri-Minet M, Reuter U, Del Río MS, Schoenen J, Schwalen S, van Oene J; TOPMAT-MIG-303 Investigators Group: Cessation versus continuation of 6-month preventive therapy with topiramate (PROMPT): a randomised, placebo-controlled clinical trial. Lancet Neurol 2007, 6:1054-62.

18. Pascual J, El Berdei Y, Gómez-Sánchez JC: How many migraine patients need prolonged ( $>$ I year) preventive treatment? Experience with topiramate. J Headache Pain 2007, 8:90-3.

FI000 Factor 3.0 Recommended Evaluated by Jean Schoenen 14 Mar 2007

19. Pascual J, Rivas MT, Leira R: Testing the combination betablocker plus topiramate in refractory migraine. Acta Neurol Scand 2007, I | 5:81-3. 